\title{
Original
}

\section{CXCR4 signaling contributes to alveolar bone resorption in Porphyromonas gingivalis-induced periodontitis in mice}

\author{
Hidekazu Nagashima'), Masamichi Shinoda'), Kuniya Honda'), Noriaki Kamio ${ }^{3)}$, \\ Akira Hasuike4), Naoyuki Sugano4), Yoshinori Arais), Shuichi Sato4), \\ and Koichi Iwata ${ }^{2}$
}
1)Division of Applied Oral Sciences, Nihon University Graduate School of Dentistry, Tokyo, Japan
2)Department of Physiology, Nihon University School of Dentistry, Tokyo, Japan
3)Department of Microbiology, Nihon University School of Dentistry, Tokyo, Japan
4)Department of Periodontology, Nihon University School of Dentistry, Tokyo, Japan
5)Nihon University School of Dentistry, Tokyo, Japan

(Received December 1, 2016; Accepted December 22, 2016)

\begin{abstract}
Periodontitis caused by bacterial infection gradually progresses accompanied by periodontal tissue destruction. As a result, teeth lose their supporting structures, and this leads to tooth exfoliation. CXC-chemokine receptor 4 (CXCR4) is known to be expressed in lymphocytes, fibroblasts and osteoclasts in periodontal tissues, suggesting that periodontal CXCR4 signaling contributes to alveolar bone resorption in the milieu of periodontitis. However, the role of CXCR4 signaling in the pathogenesis of periodontitis has remained unknown. We established a mouse model of periodontitis by inoculation of Porphyromonas gingivalis (P.g.) into a silk ligature placed around the maxillary molar. Although there was no significant difference in the mechanical sensitivity in the periodontal tissue between P.g. treatment and sham treatment during the experimental period, mechanical allodynia in the periodontal tissue was induced after gingival injection of complete Freund's adjuvant compared with that resulting from sham and P.g. treatment alone. More-
\end{abstract}

Correspondence to Dr. Masamichi Shinoda, Department of Physiology, Nihon University School of Dentistry, 1-8-13 KandaSurugadai, Chiyoda-ku, Tokyo 101-8310, Japan

Fax:+81-3-3219-8341 E-mail: shinoda.masamichi@nihon-u.ac.jp

J-STAGE Advance Publication: October 31, 2017

Color figures can be viewed in the online issue at J-STAGE.

doi.org/10.2334/josnusd.16-0830

DN/JST.JSTAGE/josnusd/16-0830 over, CXCR4 neutralization in the periodontal tissue following $P$.g. treatment enhanced periodontal inflammatory cell infiltration and depressed alveolar bone resorption. These findings suggest that periodontal CXCR4 signaling in several cell types in P.g.-induced periodontal inflammation depresses alveolar bone resorption in periodontitis. CXCR4 signaling might be a target for therapeutic intervention to prevent alveolar bone resorption in periodontitis.

Keywords: CXCR4: Porphyromonas gingivalis; periodontitis; alveolar bone resorption; micro-computed tomography.

\section{Introduction}

Periodontal diseases such as gingivitis and periodontitis are progressive multifactorial diseases caused by bacterial infection (1). A high proportion of dentate adults suffer from chronic periodontitis. For instance, in the United States, $80 \%$ of people over 65 years of age and $35 \%$ of those aged over 30 years are affected (2-4). Ordinarily, periodontal disease gradually progresses accompanied by periodontal tissue destruction $(5,6)$. This leads to loss of alveolar bone and supporting structures, and tooth exfoliation may occur (7). The key factor in the pathogenesis of periodontitis is thought to be dental plaque, a biofilm containing microorganisms or microflora, which interacts with the host and plays a significant role in the initiation 
and maintenance of periodontitis $(8,9)$. However, details of the mechanisms of periodontitis pathogenesis have yet to be clearly established $(2,9)$.

It is well known that Porphyromonas gingivalis (P.g.), a Gram-negative oral anaerobic bacterium, is the principal pathogen causing periodontitis in humans (10). The fimbriae of P.g. (P.g.-fimbriae), which serve as a major colonizing factor, bind to CXC-chemokine receptor 4 (CXCR4), and this signaling inhibits immunological reactions in periodontal tissue $(11,12)$. CXCR4 is expressed in monocytes, and P.g.-fimbriae/CXCR4 signaling activates protein kinase A (PKA) in the presence of cyclic adenosine monophosphate. P.g.-fimbriae binding to monocyte inhibits the production of nitric oxide (11). CXCR4 is also expressed in lymphocytes, fibroblasts and osteoclasts in periodontal tissues (13-16), and therefore periodontal P.g.-fimbriae/CXCR4 signaling would be expected to contribute to the pathophysiology of alveolar bone resorption in periodontitis patients. However, details of the role of periodontal CXCR4 signaling in alveolar bone resorption in the context of periodontal inflammation caused by P.g. colonization are not fully clear.

In the present study, to examine whether CXCR4 signaling is involved in alveolar bone resorption in periodontal inflammation caused by P.g. infection of periodontal tissue, we assessed changes in alveolar bone resorption using histological and micro-computed tomography $(\mathrm{mCT})$ analysis after periodontal administration of a CXCR4 neutralizing antibody in a mouse model of periodontitis induced by P.g. inoculation.

\section{Materials and Methods}

\section{Animals}

This study was performed using $39 \mathrm{C} 57 \mathrm{BL} / 6$ mice (male, 7 weeks old, 20-30 g; Japan SLC, Shizuoka, Japan). All mice were housed in individual cages under a standard light-dark cycle (lights on 7:00 to 19:00) in a temperature-controlled room $\left(23^{\circ} \mathrm{C}\right)$. The study was approved by the Animal Experimentation Committee of Nihon University (AP15D010-1) and conducted according to the guidelines of the International Association for the Study of Pain (17). All efforts were made to minimize animal suffering and the number of animals.

\section{Periodontitis model}

Under deep anesthesia induced by intraperitoneal injection of sodium pentobarbital $(50 \mathrm{mg} / \mathrm{kg})$, mice were placed on a warm mat $\left(37^{\circ} \mathrm{C}\right)$ and a ligature was placed around the maxillary right second molar using a 5-0 silk thread, while the mouth was kept open to avoid gingival tissue injury (18). On days 0,1 , and 2 after ligation, P.g. strain FDC381 $\left(200 \mu \mathrm{L}, 2 \times 10^{9}\right.$ colony-forming units in $2 \%$ carboxymethyl cellulose) was inoculated into the ligature under light anesthesia with $1.5 \%$ isoflurane (Mylan, Canonsburg, PA, USA) (P.g. group) (Fig. 1). As a sham control, mice were subjected to a sham procedure in which the mouth was kept open and $2 \%$ carboxymethyl cellulose alone was inoculated under light anesthesia (sham group). As a positive control, complete Freund's adjuvant (CFA) was injected into the gingival tissue around the maxillary right second molar under deep anesthesia (CFA group). To assess the effect of P.g. inoculation on the physical status of the mice, body weight was measured periodically during the experimental period.

\section{Periodontal mechanical sensitivity}

Mice were subjected to graded mechanical stimulation of the gingival tissue adjacent to the cervical region of the maxillary right second molar using an electronic von Frey aesthesiometer (0 to $100 \mathrm{~g}$ [cutoff, $100 \mathrm{~g}$ ], $10 \mathrm{~g} / \mathrm{s}$; Bioseb, Chaville, France) under light anesthesia with $2 \%$ isoflurane (Mylan). On day 8 after the sham, CFA or P.g. treatment, the lowest mechanical intensity required to evoke a nocifensive reflex (head withdrawal) upon mechanical stimulation was defined as the mechanical head-withdrawal reflex threshold (MHWT). The graded mechanical stimuli were applied at 1-min intervals; the MHWT was determined as the average mechanical intensity that evoked head withdrawal in response to three stimulus applications. All measurements of mechanical sensitivity in the gingival tissue were conducted under blinded conditions.

\section{Histology}

On day 14, mice were anesthetized with sodium pentobarbital $(50 \mathrm{mg} / \mathrm{kg}$, intraperitoneally) and perfused transcardially with saline followed by $4 \%$ paraformaldehyde (PFA) in $0.1 \mathrm{M}$ phosphate-buffered saline (pH 7.4). The right maxillary tissue containing the maxillary bone, maxillary second molar, and periodontal tissues were dissected out and immersed in 4\% PFA for $4 \mathrm{~h}$ at $4^{\circ} \mathrm{C}$. The maxillary tissue was then decalcified using $50 \%$ K-CX (Falma, Tokyo, Japan) for 1 day and neutralized in $5 \%$ sodium sulfate overnight. The post-fixed and decalcified maxillary tissue was soaked in $0.01 \mathrm{M}$ phosphate-buffered saline (PBS) containing 20\% sucrose for $12 \mathrm{~h}$ for cryoprotection, and then embedded in TissueTek (Sakura Finetek, Tokyo, Japan) at $-20^{\circ} \mathrm{C}$. The maxillary tissue was sectioned in the buccolingual plane on a cryostat at a thickness of $16 \mu \mathrm{m}$. The sections were 


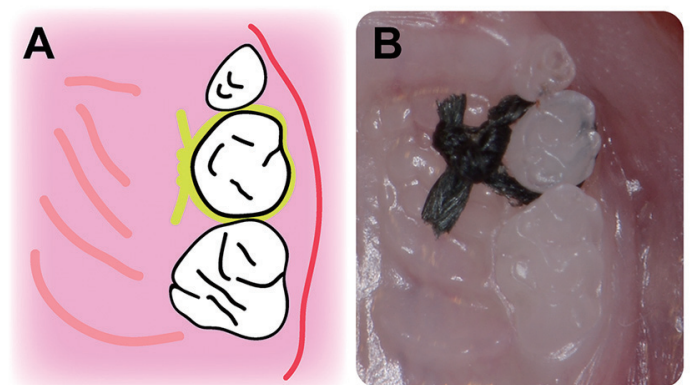

Fig. 1 Schematic illustration (A) and photograph (B) of the 5-0 silk ligature placed around the maxillary right second molar.

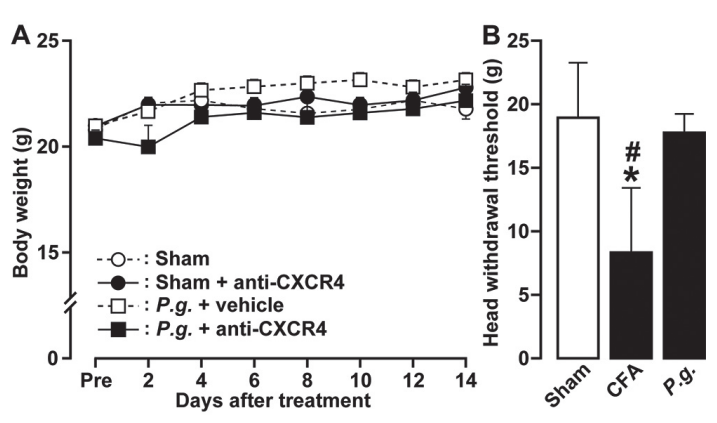

Fig. 2 Changes in body weight (A; $n=5$ or 6 in each. One-way ANOVA followed by Bonferroni's multiple comparison test) and mechanical sensitivity of gingival tissue $(\mathrm{B} ; n=3$ or 12 in each. Two-way ANOVA followed by Bonferroni's multiple comparison test for gingival mechanical sensitivity) following P.g. treatment. Data represent mean \pm SEM. *: $P<0.05$ vs. sham. ${ }^{*}: P<0.05$ vs. P.g.

$\mathrm{BV}$ in the alveolar bone around the maxillary right second molar was calculated using $\mathrm{mCT}$ analysis during the experimental period (days 0 to 14 ) as described above.

\section{Statistical analysis}

All data were expressed as the mean \pm SEM. Statistical analyses were performed using one-way and two-way repeated-measures analysis of variance (ANOVA) followed by Bonferroni's multiple comparison tests. A value of $P<0.05$ was considered to indicate statistical significance.

$1.5 \%$ isoflurane (Mylan), mice were placed on the instrument stage and $\mathrm{mCT}$ images of the alveolar bone around the maxillary right second molar were obtained under conditions in which the exposure parameters were $90 \mathrm{kV}$ and $100 \mu \mathrm{A}$. The mCT images were reconstructed using i-View software (I-View Image Center, Tokyo, Japan). Bone volume (BV) within the maxillary alveolar bone regions of interest $(1.2 \times 1.2 \times 0.6 \mathrm{~mm})$ was measured from the reconstructed $\mathrm{mCT}$ images using $\mathrm{BV}$ measuring software (Kitasenjyu Radist Dental Clinic I-View Image Center, Tokyo, Japan). The central point of the maxillary alveolar bone region of interest was defined as the lowest point of furcation between medial buccal root and the palatal root of upper first molar. The BV in the alveolar bone around the maxillary right second molar was calculated by the subtraction method (19).

\section{Drug administration}

Under light anesthesia with $2 \%$ isoflurane, anti-CXCR4 neutralizing antibody $(1 \mu \mathrm{L}, 50 \mu \mathrm{g} / \mathrm{mL}$; Cat. MAB21651; R\&D Systems, Minneapolis, MN, USA) dissolved in $0.01 \mathrm{M}$ PBS or vehicle (0.01M PBS) was administered subcutaneously into the gingival tissue for 14 successive days (day 0 to day 13) in the P.g. and sham groups. The

\section{Results \\ General condition and periodontal mechanical sensitivity}

There was no difference in body weight change between the P.g. group and the sham group (Fig. 2A), and no abnormal behavior reflecting motor deficits or mental disturbance was not observed during the experimental period (data not shown).

There was no significant difference in the MHWT between the P.g. treatment and sham groups on day 8 (Fig. 2B). The MHWT was significantly decreased on day 8 after CFA injection into the gingival tissue when compared with that after sham and P.g. treatment. No motor deficits were evident during the experimental period (data not shown).

\section{Histological examination}

Many immune cells (e.g., macrophages, lymphocytes, neutrophils and phagocytes) were detected in gingival tissue on day 14 in P.g.-treated mice administered antiCXCR4 neutralizing antibody or vehicle (Fig. 3). The number of immune cells in those mice tended to be 


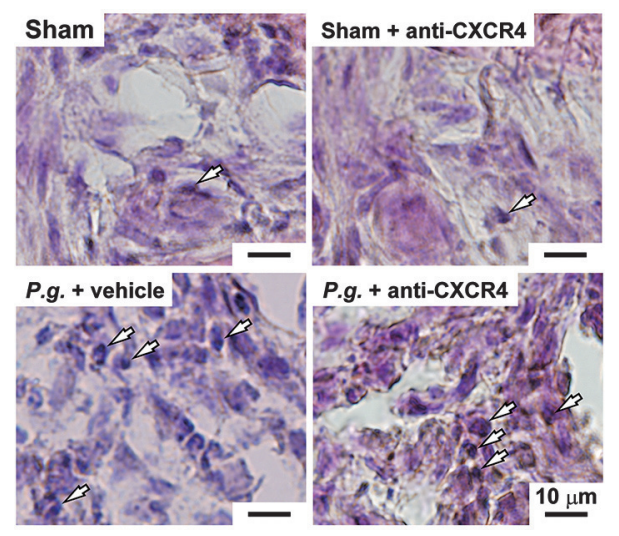

Fig. 3 Histology of periodontal tissue following sham and P.g. treatment with vehicle or administration of periodontal anti-CXCR4 neutralizing antibody. The arrows indicate inflammatory cells.

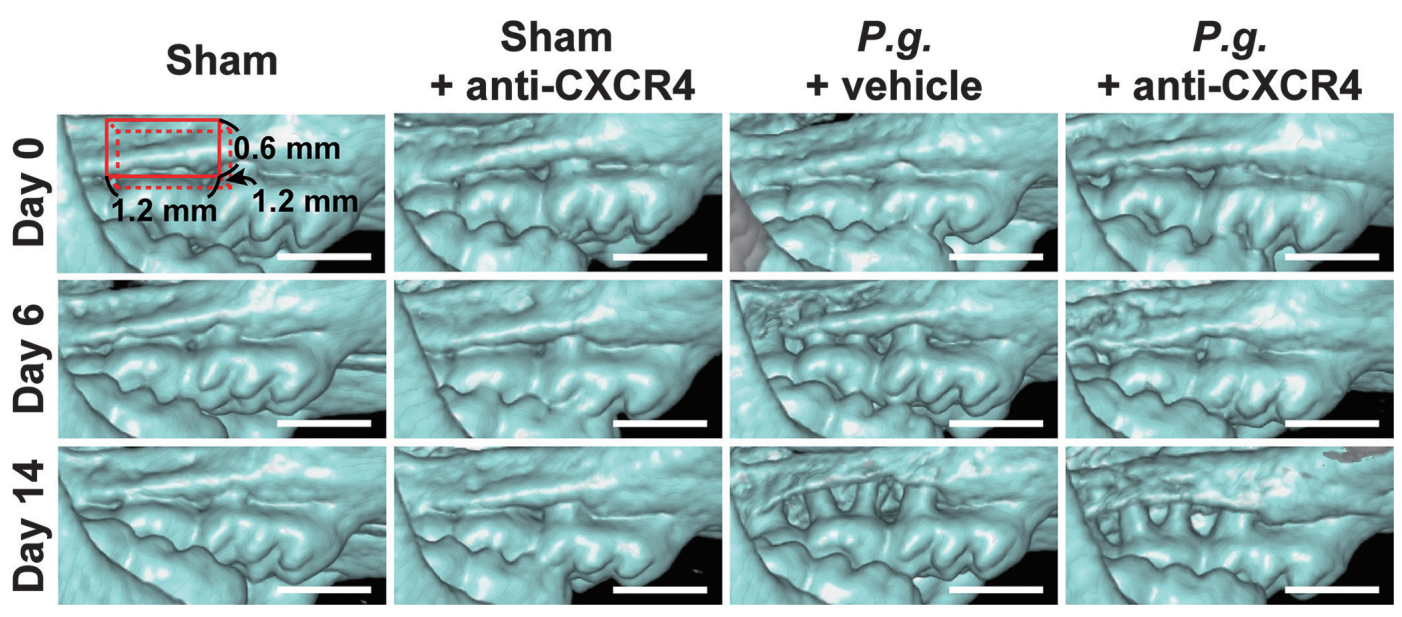

Fig. 4 Reconstructed mCT images of the maxillary alveolar bone on days 0, 6, and 14 in the sham group administered anti-CXCR4 neutralizing antibody or vehicle, and in the P.g. group administered anti-CXCR4 neutralizing antibody or vehicle. A diagram of the maxillary alveolar bone regions of interest is shown in the $\mathrm{mCT}$ images of the alveolar bone in the sham group on day 0 .

higher than in the P.g. group administered vehicle. In the sham group, a few immune cells were present on day 14 .

\section{Measurements of BV}

Typical reconstructed $\mathrm{mCT}$ images of the maxillary alveolar bone on days 0,6 , and 14 in the sham group administered anti-CXCR4 neutralizing antibody or vehicle, and in the P.g. group administered anti-CXCR4 neutralizing antibody or vehicle, are shown in Fig. 4. There were no significant differences in BV between the sham group and the group administered anti-CXCR4 neutralizing antibody. BV was significantly decreased in the $P . g$. group administered vehicle relative to the sham group between day 4 and day 14 . The decrease of BV was significantly suppressed in the P.g. group administered anti-CXCR4 neutralizing antibody relative to that in the P.g. group administered vehicle from day 6 to day
14 (Fig. 5). During the experimental period there were no abnormal findings in the reconstructed $\mathrm{mCT}$ images, such as alveolar bone fracture, necrosis and hyperplasia.

\section{Discussion}

Generally, a wide variety of chemical mediators are released from peripheral nerve endings or immune cells at sites of local inflammation. These mediators play a regulatory role in the excitability of peripheral nociceptive nerve endings, resulting in an increase of excessive afferent firing, leading to pain hypersensitivity (20). Our earlier studies using inflammatory pain models suggested that release of glutamate or calcitonin gene-related peptide locally inflamed sites ordinarily results in an increase of purinergic receptor expression in peripheral nociceptive nerve endings, creating primary afferent neuronal sensitization and subsequent orofacial pain 


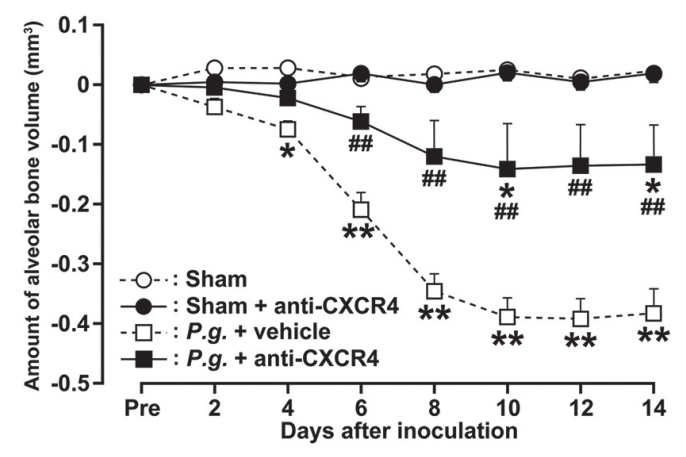

Fig. 5 Alveolar bone resorption following sham and P.g. treatment with vehicle or periodontal anti-CXCR4 neutralizing antibody. *: $P<0.05$, **: $P<0.01$ vs. sham; $: P<0.01$ vs. $P . g$. treatment with vehicle. ( $n=5$ or 6 in each. Two-way ANOVA followed by Bonferroni's multiple comparison test).

hypersensitivity $(21,22)$. Interestingly, although periodontitis is a representative inflammatory disease in of the periodontal region, patients with chronic periodontitis rarely complain of pain hypersensitivity in the affected structures (23). In the present study, although MHWT was significantly decreased in gingival tissue from day 2 after periodontal CFA injection (data not shown), there was no change in the MHWT of inflamed periodontal tissue in P.g.-treated mice showing pronounced periodontal tissue inflammation. These results indicate that this periodontal inflammation model is likely to be useful for pathophysiologic analysis of periodontitis.

In this study, significant inflammatory cell infiltration into gingival tissue was induced in P.g.-treated mice, similarly to that in CFA-treated mice. On the one hand, administration of anti-CXCR4 neutralizing antibody into the gingival tissue significantly suppressed alveolar bone resorption in the P.g. group relative to that in the $P . g$. group administered vehicle. In the sham group, administration of anti-CXCR4 neutralizing antibody into the gingival tissue did not change the volume of alveolar bone during the experimental period. A few possible mechanisms for the suppression of alveolar bone resorption in the P.g. group following CXCR4 neutralization can be considered.

CXCR4 is expressed in various cells such as fibroblasts, macrophages, osteoblasts and osteoclasts (24). Macrophage migration inhibitory factor (MIF), a proinflammatory cytokine, plays a critical role in innate immunity and has been shown to be a key mediator of inflammatory disease (25). MIF is stored in the cytoplasm of macrophages and is rapidly released in response to various stimuli (26). MIF binds to, and signals through CXCR4, which regulates mainly inflammatory cell recruitment (27). Macrophages express
CXCR4, which regulates cell migration, and inhibition of CXCR4 signaling leads to infiltration and retention of macrophages at sites of inflammation, where they release various molecules including TNF- $\alpha$ and C-C motif ligand 2 (CCL2) (28-30). TNF- $\alpha$-converting enzyme promotes osteoclast-mediated osteogenesis in periodontitis and CCL2 signaling accelerates osteoclast-mediated bone resorption (31,32). Indeed, systemic administration of a potent CXCR4 antagonist has been reported to suppress P.g.-induced alveolar bone loss, P.g. colonization and associated elevation of the total microbiota count in periodontal tissue (33). In the present study, many immune cells containing macrophages were detected in gingival tissue in P.g.-treated mice, and the number of immune cells in P.g.-treated mice administered anti-CXCR4 neutralizing antibody tended to be higher than in mice treated with vehicle. This result suggests that inhibition of periodontal CXCR4 signaling attenuated alveolar bone resorption in the P.g. group, although CXCR4 neutralization induced an increase of inflammatory cell and osteoclast infiltration. Further studies are needed to address these inconsistent results.

The expression of $\mathrm{C}-\mathrm{X}-\mathrm{C}$ motif chemokine 12 (CXCL12), known as stromal cell-derived factor-1, which acts as a ligand of CXCR4, is increased following fracture or osteotomy at the injured site or in plasma $(34,35)$. Furthermore, it is known that CXCL12/CXCR4 signaling regulates osteogenic differentiation via bone morphogenetic protein signaling and osteoblast development, indicating that this signaling in osteoblasts plays a major role in osteoblast migration to sits of bone injury (36-38). CXCR4 signaling via CXCL12 in osteoclasts enhances the differentiation, function and expression of adhesion-related molecules such as $\beta 3$ integrin, CD44 and osteopontin, indicating that the potentiating action on osteoclasts via CXCL12/CXCR4 signaling accelerates alveolar bone resorption following P.g. treatment (39). Moreover, lipopolysaccharide (LPS), one of the virulence factors of P.g., is known to play an important role in inflammatory bone loss (40). In humans, a large amount of LPS is released from periodontal pockets in which periodontal pathogens have multiplied (41). LPS stimulation of osteoclasts enhances CXCR4 expression and osteoclast migration. Also, LPS stimulation accelerates osteoclast differentiation partially via up-regulation of CXCR4 signaling (42). P.g.-induced LPS also stimulates gingival fibroblasts (HGFs) in periodontal tissues, inducing the secretion of chemical mediators such as interleukins (IL)- 6 that increase osteoclast formation and IL-8 that induces osteoclast differentiation, maturation and bone resorption (43-45). Collectively, these findings 
together with our present data suggest that the acceleration of alveolar bone resorption in periodontitis results from CXCR4 signaling in several cell types such as fibroblasts, macrophages, osteoblasts and osteoclasts in inflamed periodontal tissue.

In conclusion, CXCR4 neutralization in periodontal inflammation induced by P.g. treatment has been shown to significantly suppress alveolar bone resorption. These results suggest that CXCR4 signaling in inflamed periodontal tissue plays a significant role in alveolar bone resorption in periodontitis. In future, CXCR4 signaling in inflamed periodontal tissue might be a potential therapeutic target for management of alveolar bone resorption.

\section{Acknowledgments}

This study was performed with the aid of research grants from Sato (2014) and Uemura Funds (2014) from the Nihon University School of Dentistry, a grant from the Dental Research Center, Nihon University School of Dentistry (2016), Nihon University Multidisciplinary Research Grant for (2016) and the MEXTSupported Program for the Strategic Research Foundation at Private Universities 2013-2017. The authors declare that they have no competing interests.

\section{References}

1. Kirschneck C, Fanghänel J, Wahlmann U, Wolf M, Roldán JC, Proff P (2017) Interactive effects of periodontitis and orthodontic tooth movement on dental root resorption, tooth movement velocity and alveolar bone loss in a rat model. Ann Anat 210, 32-43.

2. Beck JD, Koch GG, Rozier RG, Tudor GE (1990) Prevalence and risk indicators for periodontal attachment loss in a population of older community-dwelling blacks and whites. J Periodontol 61, 521-528.

3. Fox CH, Jette AM, McGuire SM, Feldman HA, Douglass CW (1994) Periodontal disease among New England elders. J Periodontol 65, 676-684.

4. Albandar JM, Brunelle JA, Kingman A (1999) Destructive periodontal disease in adults 30 years of age and older in the United States, 1988-1994. J Periodontol 70, 13-29.

5. Mariotti A (1999) Dental plaque-induced gingival diseases. Ann Periodontol 4, 7-17.

6. Lakhssassi N, Elhajoui N, Lodter JP, Pineill JL, Sixou M (2005) Antimicrobial susceptibility variation of 50 anaerobic periopathogens in aggressive periodontitis: an interindividual variability study. Oral Microbiol Immunol 20, 244-252.

7. Van Dyke TE, van Winkelhoff AJ (2013) Infection and inflammatory mechanisms. J Periodontol 84, S1-7.

8. Darveau RP (2010) Periodontitis: a polymicrobial disruption of host homeostasis. Nat Rev Microbiol 8, 481-490.

9. Hajishengallis G (2014) Immuno-microbial pathogenesis of periodontitis: keystones, pathobionts, and host response. Trends Immunol 35, 3-11.
10. Pihlstrom BL, Michalowicz BS, Johnson NW (2005) Periodontal diseases. Lancet 366, 1809-1820.

11. Hajishengallis G, Wang M, Liang S, Triantafilou M, Triantafilou K (2008) Pathogen induction of CXCR4/TLR2 cross-talk impairs host defense function. Proc Natl Acad Sci U S A 105, 13532-13537.

12. Pierce DL, Nishiyama S, Liang S, Wang M, Triantafilou M, Triantafilou K et al. (2009) Host adhesive activities and virulence of novel fimbrial proteins of Porphyromonas gingivalis. Infect Immun 77, 3294-3301.

13. Havens AM, Chiu E, Taba M, Wang J, Shiozawa Y, Jung $\mathrm{Y}$ et al. (2008) Stromal-derived factor-1 $\alpha$ (CXCL12) levels increase in periodontal disease. J Periodontol 79, 845-853.

14. Shahnazari M, Chu V, Wronski TJ, Nissenson RA, Halloran BP (2013) CXCL12/CXCR4 signaling in the osteoblast regulates the mesenchymal stem cell and osteoclast lineage populations. FASEB J 27, 3505-3513.

15. Dong Y, Liu H, Zhang X, Xu F, Qin L, Cheng P et al. (2016) Inhibition of SDF-1 $\alpha /$ CXCR 4 signalling in subchondral bone attenuates post-traumatic osteoarthritis. Int J Mol Sci 17, pii: E943.

16. Sun J, Nemoto E, Hong G, Sasaki K (2017) Modulation of stromal cell-derived factor 1 alpha (SDF-1 $\alpha)$ and its receptor CXCR4 in Porphyromonas gingivalis-induced periodontal inflammation. BMC Oral Health 17, 26.

17. Zimmermann M (1983) Ethical guidelines for investigations of experimental pain in conscious animals. Pain 16, 109-110.

18. Abe T, Hajishengallis G (2013) Optimization of the ligatureinduced periodontitis model in mice. J Immunol Methods 394, 49-54.

19. Kuroki Y, Honda K, Kijima N, Wada T, Arai Y, Matsumoto N et al. (2011) In vivo morphometric analysis of inflammatory condylar changes in rat temporomandibular joint. Oral Dis 17, 499-507.

20. Muley MM, Krustev E, McDougall JJ (2016) Preclinical assessment of inflammatory pain. CNS Neurosci Ther 22, 88-101.

21. Honda K, Noma N, Shinoda M, Miyamoto M, Katagiri A, Kita D et al. (2011) Involvement of peripheral ionotropic glutamate receptors in orofacial thermal hyperalgesia in rats. Mol Pain 7, 75.

22. Yasuda M, Shinoda M, Kiyomoto M, Honda K, Suzuki A, Tamagawa $\mathrm{T}$ et al. (2012) $\mathrm{P} 2 \mathrm{X}_{3}$ receptor mediates ectopic mechanical allodynia with inflamed lower lip in mice. Neurosci Lett 528, 67-72.

23. Gaurilcikaite E, Renton T, Grant AD (2017) The paradox of painless periodontal disease. Oral Dis 23, 451-463.

24. Murdoch C (2000) CXCR4: chemokine receptor extraordinaire. Immunol Rev 177, 175-184.

25. Cooke G, Armstrong ME, Donnelly SC (2009) Macrophage migration inhibitory factor (MIF), enzymatic activity and the inflammatory response. Biofactors 35, 165-168.

26. Kim KW, Kim HR (2016) Macrophage migration inhibitory factor: a potential therapeutic target for rheumatoid arthritis. Korean J Intern Med 31, 634-642. 
27. Bernhagen J, Krohn R, Lue H, Gregory JL, Zernecke A, Koenen RR et al. (2007) MIF is a noncognate ligand of CXC chemokine receptors in inflammatory and atherogenic cell recruitment. Nat Med 13, 587-596.

28. Han YL, Li YL, Jia LX, Cheng JZ, Qi YF, Zhang HJ et al. (2012) Reciprocal interaction between macrophages and $\mathrm{T}$ cells stimulates IFN- $\gamma$ and MCP-1 production in Ang II-induced cardiac inflammation and fibrosis. PLoS One 7, e35506.

29. Turner MD, Nedjai B, Hurst T, Pennington DJ (2014) Cytokines and chemokines: at the crossroads of cell signalling and inflammatory disease. Biochim Biophys Acta 1843, 2563 2582.

30. Angsana J, Chen J, Liu L, Haller CA, Chaikof EL (2016) Efferocytosis as a regulator of macrophage chemokine receptor expression and polarization. Eur J Immunol 46, 1592-1599.

31. Kanzaki H, Makihira S, Suzuki M, Ishii T, Movila A, Hirschfeld J et al. (2016) Soluble RANKL cleaved from activated lymphocytes by TNF- $\alpha$-converting enzyme contributes to osteoclastogenesis in periodontitis. J Immunol 197, 3871 3883.

32. Khan UA, Hashimi SM, Bakr MM, Forwood MR, Morrison NA (2016) CCL2 and CCR2 are essential for the formation of osteoclasts and foreign body giant cells. J Cell Biochem 117, 382-389.

33. McIntosh ML, Hajishengallis G (2012) Inhibition of Porphyromonas gingivalis-induced periodontal bone loss by CXCR4 antagonist treatment. Mol Oral Microbiol 27, 449-457.

34. Kidd LJ, Stephens AS, Kuliwaba JS, Fazzalari NL, Wu AC, Forwood MR (2010) Temporal pattern of gene expression and histology of stress fracture healing. Bone 46, 369-378.

35. Lee DY, Cho TJ, Lee HR, Park MS, Yoo WJ, Chung CY et al. (2010) Distraction osteogenesis induces endothelial progenitor cell mobilization without inflammatory response in man. Bone 46, 673-679.

36. Hosogane N, Huang Z, Rawlins BA, Liu X, Boachie-Adjei
O, Boskey AL et al. (2010) Stromal derived factor-1 regulates bone morphogenetic protein 2-induced osteogenic differentiation of primary mesenchymal stem cells. Int J Biochem Cell Biol 42, 1132-1141.

37. Zhu W, Liang G, Huang Z, Doty SB, Boskey AL (2011) Conditional inactivation of the CXCR4 receptor in osteoprecursors reduces postnatal bone formation due to impaired osteoblast development. J Biol Chem 286, 26794-26805.

38. Yellowley C (2013) CXCL12/CXCR4 signaling and other recruitment and homing pathways in fracture repair. Bonekey Rep 2, 300 .

39. Luo T, Liu H, Feng W, Liu D, Du J, Sun J et al. (2017) Adipocytes enhance expression of osteoclast adhesion-related molecules through the CXCL12/CXCR4 signalling pathway. Cell Prolif 50, e12317.

40. O'Brien-Simpson NM, Veith PD, Dashper SG, Reynolds EC (2004) Antigens of bacteria associated with periodontitis. Periodontol 2000 35, 101-134.

41. Golub TR, Slonim DK, Tamayo P, Huard C, Gaasenbeek M, Mesirov JP et al. (1999) Molecular classification of cancer: class discovery and class prediction by gene expression monitoring. Science 286, 531-537.

42. Xing Q, de Vos P, Faas MM, Ye Q, Ren Y (2011) LPS promotes pre-osteoclast activity by up-regulating CXCR4 via TLR-4. J Dent Res 90, 157-162.

43. Roodman GD (2006) Regulation of osteoclast differentiation. Ann N Y Acad Sci 1068, 100-109.

44. Zhang Y, Li X (2015) Lipopolysaccharide-regulated production of bone sialoprotein and interleukin- 8 in human periodontal ligament fibroblasts: the role of toll-like receptors 2 and 4 and the MAPK pathway. J Periodontal Res 50, 141-151.

45. Kang W, Hu Z, Ge S (2016) Healthy and inflamed gingival fibroblasts differ in their inflammatory response to porphyromonas gingivalis lipopolysaccharide. Inflammation 39, 1842-1852. 